\title{
Corrigendum: Ultrasensitive and label-free molecular-level detection enabled by light phase control in magnetoplasmonic nanoantennas
}

Nicolò Maccaferri, Keith E. Gregorczyk, Thales V.A.G. de Oliveira, Mikko Kataja, Sebastiaan van Dijken, Zhaleh Pirzadeh, Alexandre Dmitriev, Johan Åkerman, Mato Knez \& Paolo Vavassori

Nature Communications 6:6150 doi: 10.1038/ncomms7150 (2015). Published 2 Feb 2015; Updated 18 Jun 2015

This Article contains typographical errors in the equations of the last sentence of the first paragraph of the Results section. This sentence should read 'In the present case, the spin-orbit transversally induced dipole is given by $p_{y}=\alpha_{y x} E_{x}^{0}=$ $\left[\varepsilon_{y x} \alpha_{y y} \alpha_{x x} /\left(\varepsilon_{0}-\varepsilon_{\mathrm{m}}\right)^{2}\right] E_{x}^{0}$ (considering the circular shape of our nanoantennas, $\alpha_{y y}=\alpha_{x x}$, that is, the two LPRs resonate at the same wavelength, and the expression above can be simplified to $\left.p_{y}=\alpha_{y x} E_{x}^{0}=\left[\varepsilon_{y x} \alpha_{x x}^{2} /\left(\varepsilon_{0}-\varepsilon_{\mathrm{m}}\right)^{2}\right] E_{x}^{0}\right)$.' 